\title{
Evaluation of Garlic-Resistance in E. coli (strain K12)
}

\author{
Pranav Kumar' ${ }^{1}$ Joshua Garcia'1, and Heather Hill' \\ ${ }^{1}$ Carnegie Vanguard High School, Houston, TX
}

\section{$\underline{\text { ABSTRACT }}$}

Antibiotic resistance, perpetuated through overuse of antibiotics, is rendering current antibiotics ineffective, minimizing the benefits of the Antibiotic Era. Despite research on garlic-based treatments as a safe and effective alternative to conventional antibiotics, there is a lack of understanding if bacteria can grow resistant to garlic itself. However, given that garlic is an effective bactericidal agent and can disrupt biofilm production, an important factor in the ability of bacteria to resist antibiotics, garlic may be immune to antibiotic resistance. Thus, this study seeks to answer the following question: will E. coli (K12) display increased resistance (lower susceptibility) to a garlic-based treatment, as evaluated by a disk diffusion test, over two generations? An agar diffusion test was performed on two duplicate groups of E. coli with identical garlic impregnated disks. This test was then repeated on cultures obtained from the first test using an identical garlic-based treatment. The minimum distance of bacteria colonies from the disk was measured for both generations and compared to ascertain if the E. coli showed increased resistance to the treatment. The first generation of E. coli for both group A and group B had colonies with a minimum distance of 5 millimeters from the disk. The second generation of E. coli for both group A and group B had colonies in contact with the disk, leading to a minimum distance of 0 millimeters. These results indicate that E. coli displayed increased resistance to a garlic-based treatment over the course of two generations.

\section{Introduction}

Antibiotics were a revolutionary, field-defining discovery that had massive, positive, implications on human health and well-being. Indeed, the shift from the pre-Antibiotic era of the early 1900s to the modern Antibiotic era saw a shift away from deaths by communicable diseases, thereby playing a significant role in the surge in life expectancy from 47 to 78.8 in the USA (Adedeji, 2016). Problematically, antibiotic resistance, a phenomenon brought forth by widespread usage of antibiotics, poses an existential threat to all the benefits of the Antibiotic Era. In reaction to this, scientists are looking to substances that are naturally antimicrobial and do not require human intervention to be made, such as garlic, turmeric, and ginger. Garlic, in particular, holds key interest to researchers because numerous empirical studies have proven its efficacy at terminating bacterial life. For example, a study examining the effect of garlic on bacteria isolated from the Hawassa Referral Hospital, Ethiopia, (Abiv et al, 2016) and an independent study evaluating the effect of allicin, the active ingredient in garlic, and its analogues on bacteria (Leontiev et al, 2018) both found garlic, or its active compound, to be a bactericidal agent. The effect of garlic on previously antibiotic resistant bacteria is a topic that has been researched extensively; however, there have been no studies looking at the development of new antibiotic resistance to garlic. This begs a simple but extremely important and unanswered question of if bacteria can grow resistant to garlic itself. Despite collective optimism from the scientific community regarding garlic as a means to counter the dangerous spread of antibiotic resistance, there is a lack of understanding behind garlic's antibiotic properties in the context of garlic resistance. Since there is no definitive explanation behind whether or not microbes can grow resistant to a garlic-based treatment, this gap in understanding makes evaluating the issue of resistance to garlic a very compelling direction for research. This direction needs to be explored quickly, because as long as ambiguity exists in the context of treating antibiotic resistance, lives will be lost; Aslam et al quantify that in the "worst-case scenario" for antibiotic resistance, the world population would be hugely affected: as of the year 2050 , 
about 444 million people would succumb to infections and birthrates would rapidly decline (Aslam et al, 2018). A study that explores bacterial resistance to garlic would represent an important contribution in the global fight against antibiotic resistance by evaluating humanity's figurative spearhead of solvency. This project seeks to answer the following question: will E. coli (strain K12) display increased resistance (lower susceptibility) to a garlic-based treatment, as evaluated by a disk diffusion test, over two generations? An important assumption would be that garlic is a bactericidal agent and will display these characteristics in a lab setting. Fortunately, contemporary literature strongly indicates this to be true (Abiy et al, 2016). Evaluating E. coli's ability to become resistant to garlic is the first step towards determining if garlic-resistance is a veritable threat to garlic-based treatments.

\section{Literature Review}

Antibiotic resistance is a complex phenomenon borne of many different factors. One such factor is mutations due to over-prescription, or over usage, of current antibiotic medications. For example, an annual review by the CDC in 2017 found that almost $30 \%$ of the antibiotics prescribed in outpatient clinics were deemed unnecessary (CDC, 2017). The paper went on to imply that this quantification of magnitude likely does not do true justice to the root problem because even when there was need for antibiotics, prescribers tended to favor less effective medicines that carried more risk than targeted first line medications, as recommended by national guidelines. This statistic is important in the context of the mechanism behind how bacteria gain resistance. Ultimately, acquisition of resistance comes down to an evolutionary arms race between humans and pathogens; humans find newer ways to neutralize bacteria and bacteria develop newer ways to survive, often through random mutations that leave human antibiotics obsolete (Woodford et al, 2007). Problematically, when the figurative dust clears, the most resistant bacteria are left to reproduce and perpetuate their resistance genes via future generations. This means that when prescribers use ineffective or unnecessary antibiotics, as premised by the CDC as of 2017, the minute surviving populations can display resistance which then propagates itself to a massive scale through rapid reproduction through the mechanism identified by Woodford et al. In this context, if this project finds that $E$. coli is able to show increased resistance to a garlic-based treatment, this method would be the most likely way the bacteria gained resistance. If this is the case, then the analysis by the CDC on the spread of antibiotic resistance will also need to be considered in the context of garlic-based treatments. Regardless, acquisition of antibiotic resistance is further compounded by a nuanced but powerful tool that pathogens behold biofilm production.

Biofilms are a vast matrix of microorganisms that have attached with one another to form a syntrophic consortium. Relevantly, biofilm production can theoretically provide inherent resistance from antibacterial medications via poor antibiotic penetration and adaptive stress response (Steward, 2004). This claim has also been proven true empirically; the unique persistence of, for example, staphylococcal infections related to foreign bodies is due to biofilm formation (Høiby, 2010). Perhaps anticipating this implication, Steward concludes that "disabling biofilm resistance may enhance the ability of existing antibiotics to clear infections involving biofilms that are refractory to current treatments." Overall, biofilms prove to be troublesome to resolve when using antibiotics.

The idea of intrinsic phenotypic resistance to antibiotics is troublesome enough - but biofilm growth is also associated with an increased level of mutations as well as with quorum sensing-regulated mechanisms (Høiby, 2010). Quorum-sensing is an intercellular communication device that allows bacteria to synchronize the gene expression of the group, and thus, act in unison (Wai-Leung, 2015). This indicates that the resistance gene can spread from resistant bacteria to normal bacteria via quorum sensing mechanisms facilitated by biofilm. The implication is that bacteria are leveraging genotypic resistance to modern medicine - and then spreading it throughout their populations. As a result, more and more bacteria stand to grow resistant to antibiotics through this perpetuation of the resistance gene. By identifying a way to disrupt the formation of biofilm, or a way to disrupt it once formed, scientists will be able to address one of the ways bacteria are able to show both phenotypic and genotypic resistance to antibiotics. This is where garlic, and research related to garlic, enters the scientific spotlight. 
Although there is little evidence to suggest that garlic suppresses acquisition of antibiotic resistance, evidence suggests garlic is capable of suppressing biofilm production and quorum sensing. First, numerous studies have empirically found garlic to be effective in killing bacteria. Allicin, the active ingredient in garlic, has been shown to drive this antibacterial effect (Wallock Richards et al, 2014). With this in mind, Abiy et al further examined garlic and found it to be highly effective in killing E. coli and Staphylococcus aureus. These results strongly indicate garlic to be able to neutralize a wide range of bacteria, including both gram positive and gram-negative bacteria. Confirming this analysis, Leontiev et al examined the effects of garlic's active ingredient and affirm that allicin shows broad antimicrobial activities against gram-positive bacteria, gram negative bacteria, and even bacteria resistant to other antibiotics. In particular relevance to antibiotic resistance, garlic (and its active ingredient allicin) has also been shown to empirically disrupt the formation of biofilm - one study published in the prestigious Journal of Microbiology found that garlic extracts could reduce $62.72 \%$ of biofilm formation of the bacteria that were tested (Mohsenipour et al, 2015). This supports a more recent study that explored the delivery of garlic extracts through nanoparticles and found it efficient in penetrating and disrupting well established methicillin-resistant Staphylococcus aureus (MRSA) biofilms (Girish et al, 2019). These findings are critical because, as identified earlier in this paper, biofilms (and, by extension, quorum sensing) are a major focus point in the fight against antibiotic resistance. The findings of these two experiments strongly suggests that garlic, on top of being bactericidal, is able to address the issues of biofilm production and possibly address quorum sensing in addition. This revelation only further warrants researchers' enthusiasm in researching garlic and allicin for combating antibiotic resistance. This analysis implies that garlic may be inherently resistant to the phenomenon of antibiotic resistance - in other words, due to garlic's ability to terminate bacteria and disrupt the biofilm production and quorum sensing of any remaining bacteria, bacteria might not theoretically be able to gain resistance to a garlic-based treatment in the first place. Thus, garlic seems to prove itself as a poster-child for the next generation replacement for antibiotics - it shows broad-spectrum antibiotic effects against a variety of species of bacteria, including bacteria that was already resistant to other antibiotics. However, the unique importance of garlic's role in the fight against antibiotic resistance also makes the issue of determining whether or not bacteria can grow resistant to garlic extracts in actuality all the more important. Theoretical notions aside, knowing whether or not bacteria can become garlic-resistant in practice may prove to be a key distinction that can protect it from being hindered by the same issues that are plaguing contemporary medicine. If this is the case, then issues like those pinpointed in the 2017 CDC report detailing misuse of antibiotics must be addressed and applied to future garlic based treatments to prevent one of humanity's biggest spearhead against antibiotic resistance from falling prey to the very same phenomenon felling current medicine.

\section{Methods}

This study warranted a research design that experimentally determined if Escherichia coli (strain K12) will display higher resistance (lower susceptibility) to pressed garlic extract, as measured by an agar diffusion test, over 2 generations. First, Escherichia coli (strain K12) was chosen for this research project because, as Professor Cooper explains in The Cell: A Molecular Approach. 2nd edition, the high division rate of this strain makes it ideal for studies looking into antibiotic resistance due to the ease of isolating garlic-resistant mutants. In addition, Escherichia coli can form biofilm, which makes this study more applicable to other bacteria that also form biofilm, which is important because, as mentioned previously, biofilms play a crucial role in bacterial resistance. The fact that the K12 strain is non-pathogenic makes the study safer for the experimenter. An agar diffusion test (specifically, a disk diffusion test) was chosen because this type of test is of the most commonly used types in literature when evaluating the antibiotic susceptibility of microbes (Hudzicki, 2009) and is ideal for visually affirming or negating increasing resistance or susceptibility to a substance. Thus, an agar diffusion test will make this research most applicable to current advances in microbiology. First, Luria-Bertani (LB) broth and agar was prepared by sterilizing deionized water. Afterwards, agar and nutrient powder were added to the sterile water (1.5:100 ratio of agar to water by mass and 2:100 ratio of nutrient to water by mass, which is the typical recipe for LB media). LB media was chosen because it allows for an ideal 
reproducibility of the physiological state of the bacteria (Sezenov et al, 2007). This is relevant to this procedure because the purpose is to isolate garlic-resistant bacteria - and, in the case that garlic-resistant bacteria does grow, this resistance will need to be reproduced so that a difference between the original stock bacteria and novel resistant bacteria can be sufficiently evaluated in the second generation. Garlic was crushed using a garlic press and liquid was extracted through filtration. Sterile filter paper disks (typical of an agar disk diffusion test) were saturated with the garlic extract. For this procedure, Porcelain garlic was used due to its characteristically high allicin content (which is important because allicin has been identified as the active ingredient behind the antibiotic effect). It is also one of the most common type of garlic found in the grocery store. This experiment sought saturation of paper disks instead of a pre-determined amount of liquid in order to ensure consistency between trials by minimizing the chance for human error via measurement.

A slant-culture of $E$. coli $\mathrm{K} 12$ was obtained from Carolina Biological - the company ensured a quality product of high purity. Bacteria from the slant culture was transferred onto a prepared agar plate and allowed to reproduce. These bacteria acted as the standard, stock $E$. coli $\mathrm{K} 12$ for generation one of this experiment. Using a sterile inoculating loop, a single colony of bacteria from the stock $E$. coli $\mathrm{K} 12$ was added to a sterile test-tube with LB broth and incubated at 36 degrees centigrade (standard temperature for E. coli incubation) for a period of 24 hours (standard time of incubation). After 24 hours, a clean agar plate was inoculated using the $E$. coli broth via a micropipette. A spreader was used to evenly spread the bacteria and then a garlic extract saturated paper disc was placed in the center of the plate. This is important because the bacteria must be evenly spread to ensure even exposure to the garlic extract as it diffuses from the paper disk into the agar. The usage of a micropipette allows for a constant amount of broth - and by extension, a constant amount of bacteria - to ensure replicability. This system was then incubated at 36 degrees centigrade for a period of 24 hours (same temperature and time to ensure consistency and replicability). In addition, a control consisting of a blank paper plate in an inoculated agar plate incubated simultaneously ensured that only the prescribed treatment (garlic extract) was influencing the bacteria. A zone of inhibition in the control group would indicate contamination of discs with a compound other than garlic, rendering the experiment unreliable. A negative control group was also used - this petri dish was not inoculated with any bacteria. A disk without treatment was placed in its center and was incubated with the rest of the experiment. Growth of bacteria on the negative control would indicate microbial contamination of agar or disks and would render the experiment unreliable. After 24 hours, the resulting zone of inhibition for generation one was measured. This was done by finding the colony closest to the disk and measuring the distance between the bacteria colony and the filter paper using a ruler. Using a sterile inoculating loop, a single colony of bacteria closest to the paper disk in terms of distance was introduced into a new test tube containing sterile LB broth. By picking the bacteria closest to the paper ring (which is growing on the part of the agar plate with the highest concentration of garlic extract), the procedure seeks to isolate the subgroup of bacteria that display the highest level of resistance to the garlic extract. This is then incubated at 36 degrees centigrade for 24 hours to allow the second generation of $E$. coli to grow within the test tube. After 24 hours, a fresh agar plate is inoculated using the broth from this test tube. A constant and consistent spread of bacteria is ensured by the usage of a micropipette and sterile spreader. A garlic disk identical to that used in generation one is placed onto the center. This system is then incubated once again at 36 degrees centigrade and for a period of 24 hours to allow the bacteria on the agar to grow. The new ring of inhibition produced by the second batch of $E$. coli is then measured (via ruler) at the location where the radius of the zone of inhibition is the smallest. This is then compared to the zone of inhibition produced by the original, stock, $E$. coli (generation 1). This entire procedure was repeated with a total of two groups of E. coli K12 (group A and group $\mathrm{B}$ - both groups were identical). 


\section{Results and Analysis}

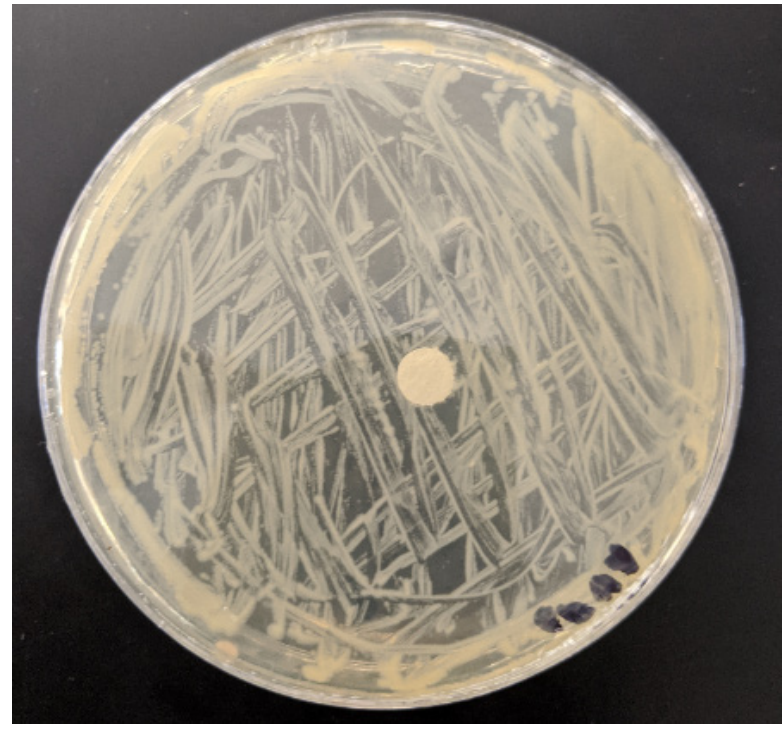

Figure 1. Petri-dish with E. coli and disk without treatment (control).

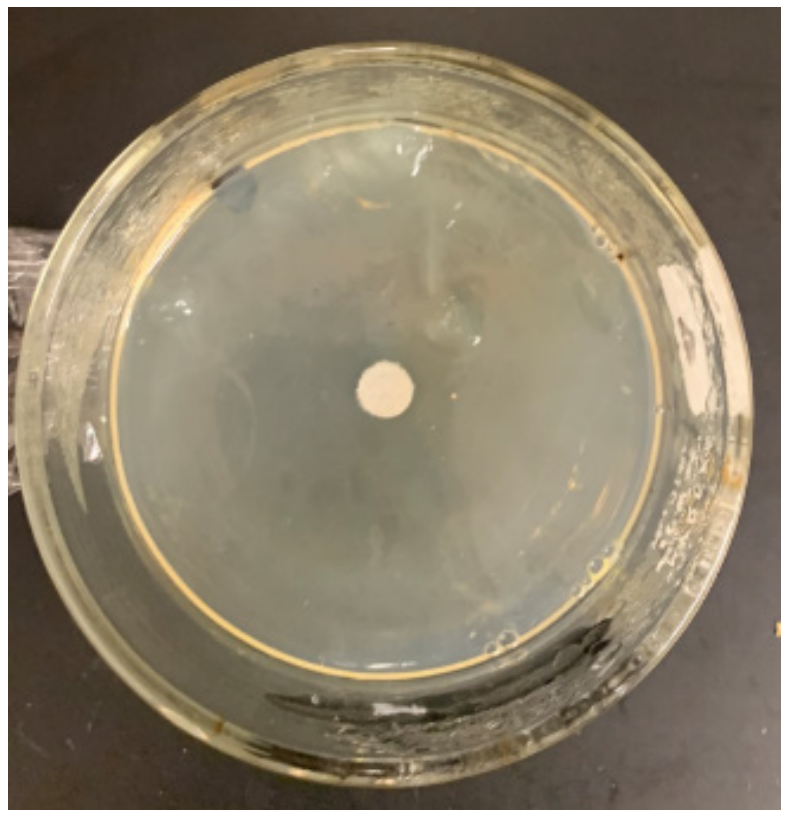

Figure 2. Petri-dish without E. coli and disk without treatment (negative control). 


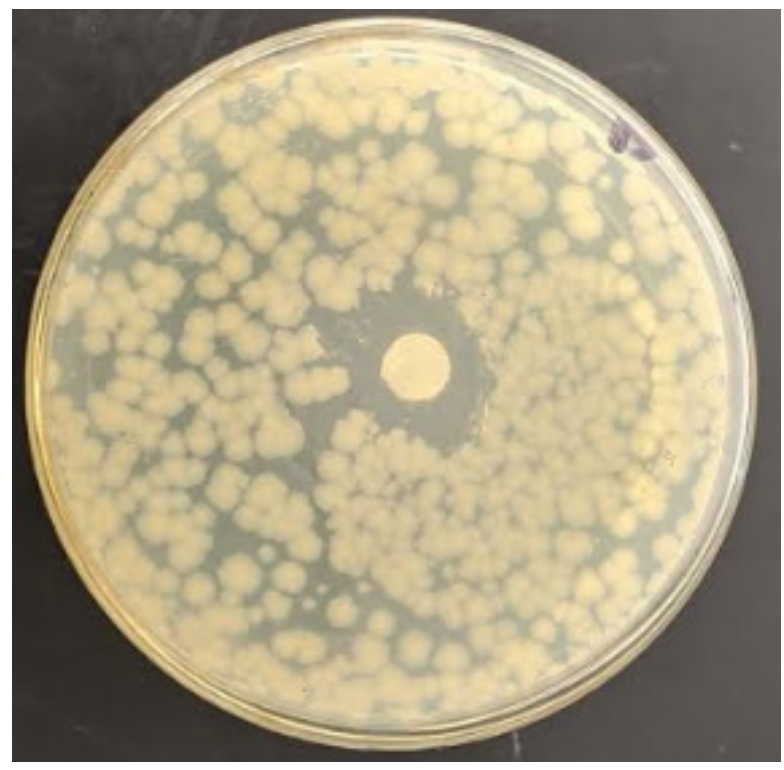

Figure 3. First generation of group A (E. coli treated with garlic).

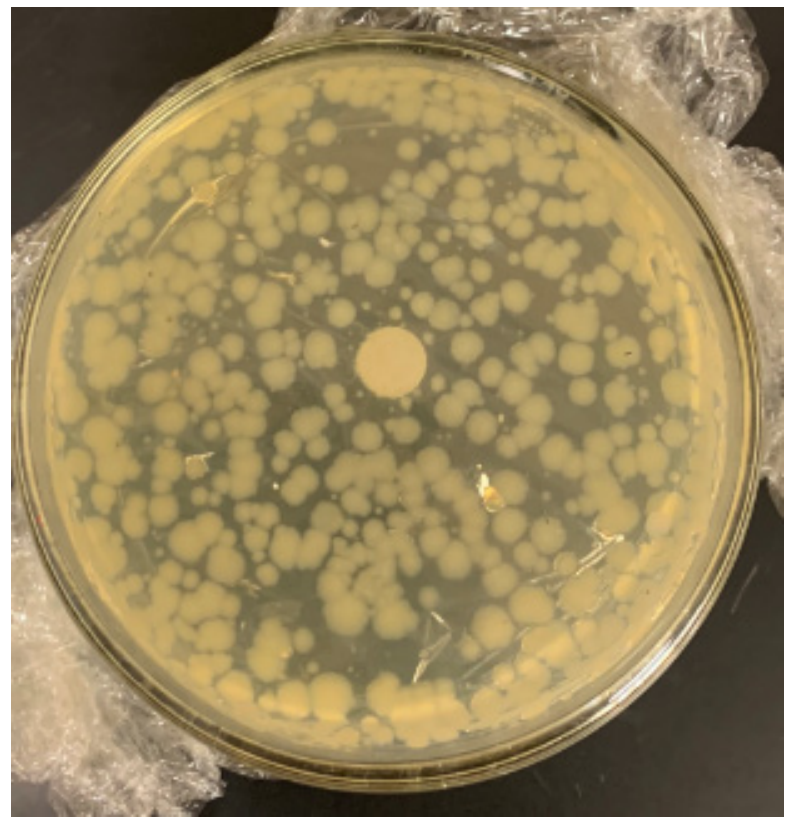

Figure 4. Second generation of group A (E. coli treated with garlic). 


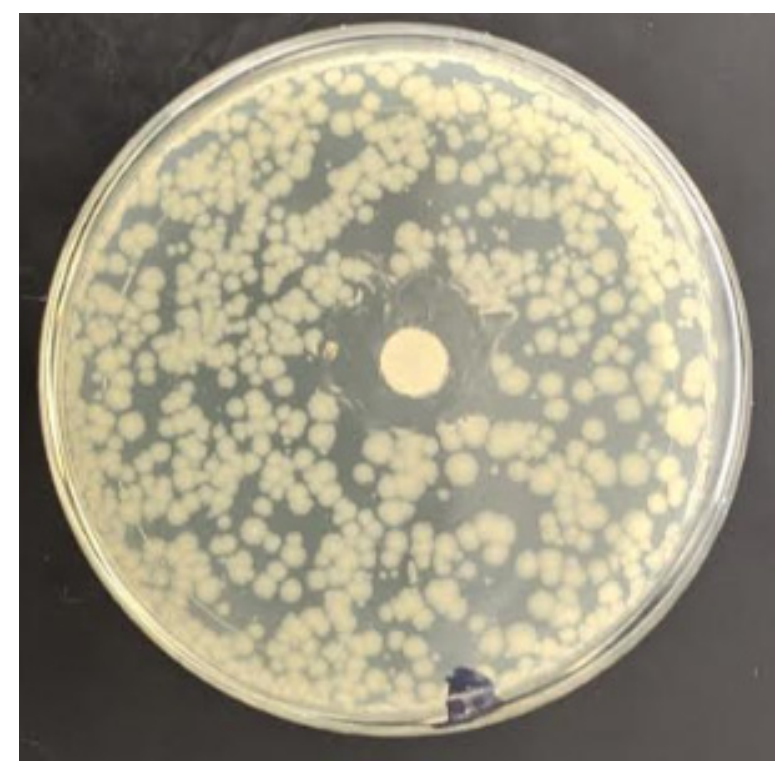

Figure 5. First generation of group B (E. coli treated with garlic).

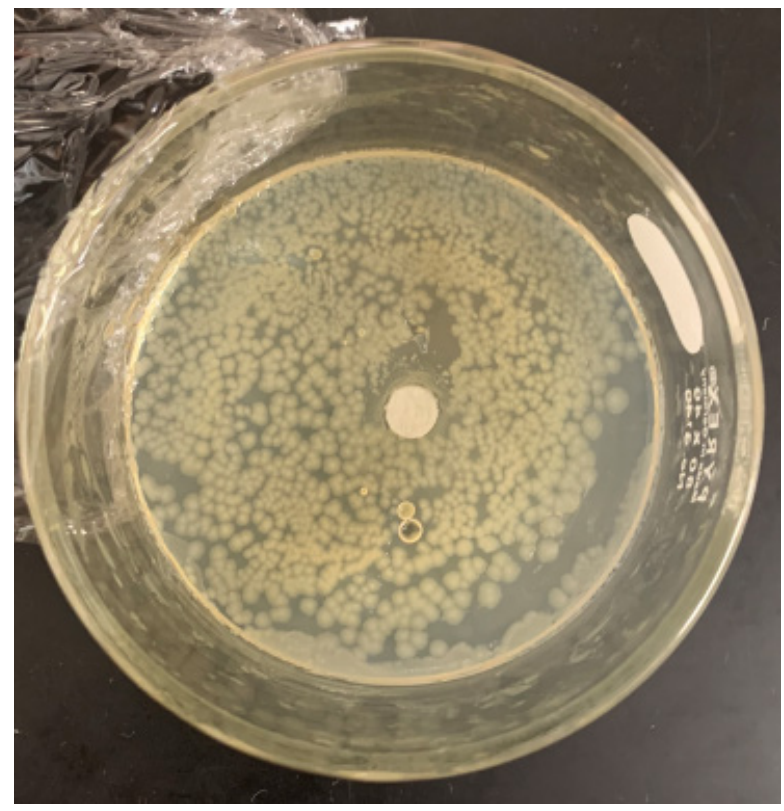

Figure 6. Second generation of group B (E. coli treated with garlic).

Note: Although this disk has irregular areas where bacteria did not grow, there are nevertheless bacteria colonies in contact with the disk, indicating the existence of resistant colonies. 
Table 1. Distance from garlic-infused disk to closest E.coli colony.

\begin{tabular}{c|c|c|}
\multicolumn{2}{c}{ Generation 1 } & Generation 2 \\
\hline \multirow{2}{*}{$\begin{array}{c}\text { Group A } \\
\text { Group B }\end{array}$} & $5 \mathrm{~mm}$ & $0 \mathrm{~mm}$ \\
\cline { 2 - 3 } & $5 \mathrm{~mm}$ & $0 \mathrm{~mm}$ \\
\cline { 2 - 3 }
\end{tabular}

Note: The measurement started at the closest colony to the paper disk and ended at the border of the paper disk. For colonies that were in contact with the paper disk, the distance was recorded as zero. This happened for the second generation of both group A and group B.

As per the figures provided, the control group (Figure 1) had no zone of inhibition, as expected, and the negative control group (figure 2) had no growth whatsoever, as expected. This indicates that any contamination (microbial or otherwise) of the agar or disks was improbable. The first generation of $E$. coli in group A and group B both had very clear zones of inhibition (refer to figures 3 and 5). As per Table 1, the colonies closest to the paper disk were 5 millimeters away from the disk for both groups. This was because as the garlic extract diffuses through the agar, its concentration within the agar reduces as the distance from the disk increases. Until a certain distance from the disk, the $E$. coli in generation 1 for both group A and group B were unable to tolerate the concentration of the garlic extract, resulting in a zone of inhibition. The border of this ring-like formation consists of the maximal concentration of garlic extract that the bacteria was able to tolerate. Measurement via ruler determined that the closest E. coli colonies in group A and group B were both half a centimeter away (5 millimeters) from the paper disk.

The second generation of bacteria was composed of bacteria from the edge of the zone of inhibition from generation one. This means that if the zone of inhibition in the second generation is greater than or equal to that in the first generation, the E. coli was not able to show any additional resistance to garlic in this experiment because the second generation was able to tolerate a maximum of either an equal or lesser concentration of garlic extract. However, in generation 2, both group A and group B had colonies that were in contact with the disk (refer to figures 4 and 6); by evaluating the distance between a colony and a paper disk at the point where the colonies were closest to the disk, the zones of inhibitions for generation two were evaluated as zero and therefore smaller than those for generation one (refer to Table 1). This meant that certain E. coli colonies in generation two were able to tolerate a higher maximum concentration of garlic than in generation one. Since all the disks in the experiment were functionally identical, this strongly indicates that the most garlic-resistant $E$. coli in generation two were more resistant to garlic than the most garlic-resistant $E$. coli in generation one. On net, the $E$. coli became more garlic-resistant over two generations, showing reduced susceptibility due to a diminishing zone of inhibition. Overall, these findings support the conclusion that, on net, E. coli is able to become more resistant to a garlic-based treatment within a petri-dish environment.

\section{Discussion}

Much of the findings from generation one was anticipated. The finding that the plates in generation one had clear zones of inhibition, as expected, attested to the bactericidal effect of garlic extract, as indicated by the findings of Abiy et al and Leontiev, both of whom experimentally showed the antibacterial properties of garlic (or allicin and analogues, in the case of the latter researcher). However, the findings from generation two were rather unexpected. Recall the research of Høiby and Steward, both of whom independently indicated that disruption of biofilm production is key to combating resistance. In this context, as well as in the context of the findings of Mohsenipour et al, which indicate that garlic extracts do, in fact, disrupt biofilm production, it is not unreasonable to be surprised when $E$. coli was able to show such drastic an increase in resistance to that very substance in this experiment in both of the tested groups, despite evidence suggesting that key factors in the development of resistance should have been disrupted. A possible explanation for this occurring is that the biofilm disruption effect does not prevent bacteria from developing random mutations that may grant resistance to garlic, which means that surviving mutant $E$. coli was able to reproduce and grow resistant to the treatment. This explanation would go hand-in-hand with the findings of the CDC report from 
2017, which identified this exact mechanism as a cause of worry in the status quo because, as per the analysis by Woodford et al, this happens to be the primary method of acquisition of resistance within bacteria. Since this study had no way of evaluating the degree of biofilm disruption, or if this disruption even happened in the first place, there is no way of knowing if interactions with the biofilm contributed to the results in any way. However, in the face of evidence suggesting that garlic should suppress key mechanisms behind acquisition of resistance (specifically biofilm and quorum sensing, both of which are key antibiotic resistant factors), the results from this study are surprising. Although more research needs to be done to evaluate if these results apply to other strains of $E$. coli and other species of bacteria, this project finds that Escherichia coli (strain K12) will display greater resistance (lower susceptibility) to pressed garlic extract, as measured by an agar diffusion test, over 2 generations.

This study had a key limitation in that it only finds that a specific non-antibiotic resistant strain (K12) of $E$. coli was able to show higher resistance to garlic over two generations. However, there are many species of bacteria, each having different biological mechanizations and a different way of responding to environmental stresses. Therefore, there is no guarantee that other strains of $E$. coli or other species of bacteria would react to a garlic-based treatment in an identical manner to the specie used in this project (E. coli K12). Given that Leontiev explores the interactions between garlic and antibiotic resistance bacteria and shows garlic having great potential in combating these menaces within one generation, progression of Leontiev's research is a next step to address the limitations of this project; an experiment that evaluates if antibiotic resistant bacteria (such as those used in Leontiev's study) will also show similar results and develop resistance to garlic over two (or possibly multiple) generations will be key in evaluating bacteria's potential for developing resistance to garlic-based treatments. This would be especially useful for researchers interested in developing garlic-based treatments to combat bacteria that are already resistant to conventional antibiotics. There are also important directions for future research regarding studies exploring the link between biofilm and antibiotic resistance. The findings from this study are limited in that they are not implicative of the interactions between bacteria biofilm, which is a key factor in antibiotic resistance, and garlic extract. However, the results from this project suggested that the biofilm-suppressing qualities of garlic, as identified by Mohsenipour et al, did little to prevent $E$. coli from gaining resistance to the garlic-based treatment. The next step for progression of research is to evaluate whether or not garlic-biofilm interactions are significant in the context of bacteria gaining resistance to garlic. This then needs to be cross applied to antibiotic resistant bacteria that also form biofilm, such as MRSA biofilms (Girish et al, 2019), to explore importance of biofilm production in antibiotic resistant bacteria, as compared to that in non-resistant bacteria within the process of gaining resistance to garlic-based treatments; the Høiby analysis is key here - recall his finding that the unique persistence of MRSA infection is due to biofilm production. It may be possible that this would apply to garlic-based treatments in addition to conventional treatments, although there is no way of knowing without future procedures. Because antibiotic resistant bacteria are fundamentally different from normal bacteria, a comparison of interactions between the biofilm of normal bacteria and garlic versus the interactions between the biofilm of antibiotic resistant bacteria and garlic may identify a difference, although it is difficult to predict what such a study might find. Overall, the best way to fan the spark lit by this project is to repeat the procedure with more species of bacteria, especially antibiotic resistant bacteria, to evaluate the true scope of potential garlic resistance in bacteria.

\section{References}

Abiy, E., \& Berhe, A. (2016, November 14). Anti-Bacterial Effect of Garlic (Allium sativum) against Clinical Isolates of Staphylococcus aureus and Escherichia coli from Patients Attending Hawassa Referral Hospital, Ethiopia. Retrieved from http://infectious-diseasesand-treatment.imedpub.com/antibacterial-effect-of-garlic-allium-sativumagainst-clinicalisolates-of-staphylococcusaureus-and-escherichia-coli-from-patientsa.php?aid=17777.

Adedeji, W. A. (2016, December). THE TREASURE CALLED ANTIBIOTICS. Retrieved October 14, 2019, from https://www.ncbi.nlm.nih.gov/pmc/articles/PMC5354621/. 
Aslam, B., Wang, W., Arshad, M. I., Khurshid, M., Muzammil, S., Rasool, M. H., ... Baloch, Z. (2018, October 10). Antibiotic resistance: a rundown of a global crisis. Retrieved from https://www.ncbi.nlm.nih.gov/pmc/articles/PMC6188119/.

CDC. Antibiotic Use in the United States, 2017: Progress and Opportunities. Atlanta, GA: US Department of Health and Human Services, CDC; 2017

Cooper GM. The Cell: A Molecular Approach. 2nd edition. Sunderland (MA): Sinauer Associates; 2000. Cells As Experimental Models. Available from: https://www.ncbi.nlm.nih.gov/books/NBK9917/

Donlan, and Rodney M. "Biofilm Formation: A Clinically Relevant Microbiological Process." OUP Academic, Oxford University Press, 15 Oct. 2001, https://academic.oup.com/cid/article/33/8/1387/347551.

Girish, Vallerinteavide Mavelli, et al. "Anti-Biofilm Activity of Garlic Extract Loaded Nanoparticles.” Nanomedicine : Nanotechnology, Biology, and Medicine, U.S. National Library of Medicine, Aug. 2019, https://www.ncbi.nlm.nih.gov/pubmed/31085344.

Høiby, Niels, et al. "Antibiotic Resistance of Bacterial Biofilms.” International Journal of Antimicrobial Agents, Elsevier, 10 Feb. 2010, https://www.sciencedirect.com/science/article/abs/pii/S0924857910000099?via=ihub

Leontiev, R., Hohaus, N., Jacob, C., Gruhlke, M. C. H., \& Slusarenko, A. J. (2018, April 30). A Comparison of the Antibacterial and Antifungal Activities of Thiosulfinate Analogues of Allicin. Retrieved from https://www.ncbi.nlm.nih.gov/pubmed/29712980.

Mohsenipour, Zeinab, and Mehdi Hassanshahian. "The Effects of Allium Sativum Extracts on Biofilm Formation and Activities of Six Pathogenic Bacteria.” Jundishapur Journal of Microbiology, Kowsar, 25 Aug. 2015, https://www.ncbi.nlm.nih.gov/pmc/articles/PMC4600595/.

Sezonov, Guennadi, et al. "Escherichia Coli Physiology in Luria-Bertani Broth." Journal of Bacteriology, American Society for Microbiology (ASM), Dec. 2007, www.ncbi.nlm.nih.gov/pmc/articles/PMC2168924/.

Stewart, Philip S. "Mechanisms of Antibiotic Resistance in Bacterial Biofilms." International Journal of Medical Microbiology, Urban \& Fischer, 10 Nov. 2004, https://www.sciencedirect.com/science/article/abs/pii/S1438422104700909.

Ventola, C. L. (2015, April). The antibiotic resistance crisis: part 1: causes and threats. Retrieved from https://www.ncbi.nlm.nih.gov/pmc/articles/PMC4378521/.

Wai-Leung, and Bonnie L Bassler. "Bacterial Quorum-Sensing Network Architectures." Annual Review of Genetics, U.S. National Library of Medicine, 2015, https://www.ncbi.nlm.nih.gov/pmc/articles/PMC4313539/.

Wallock-Richards, D., Doherty, C. J., Doherty, L., Clarke, D. J., Place, M., Govan, J. R. W., \& Campopiano, D. J. (2014, December 1). Garlic revisited: antimicrobial activity of allicin-containing garlic extracts against Burkholderia cepacia complex. Retrieved from https://www.ncbi.nlm.nih.gov/pmc/articles/PMC4249831/.

Woodford, N., \& Ellington, M. J. (2007, January). The Emergence of Antibiotic Resistance by Mutation. Retrieved from https://pubmed.ncbi.nlm.nih.gov/17184282 